\title{
Usefulness of Photodynamic Therapy in the Management of Early Central Lung Cancer: A Report of Three Cases
}

${ }^{1}$ Division of Pulmonary and Critical Care Medicine, Departments of Medicine, ${ }^{2}$ Department of Pathology, Samsung Medical Center, Sungkyunkwan University School of Medicine, Seoul, Korea

Jong Hoo Lee, M.D. ${ }^{1}$, Kyeongman Jeon, M.D. ${ }^{1}$, Won-Jung Koh, M.D. ${ }^{1}$, Gee Young Suh, M.D. ${ }^{1}$, Man Pyo Chung, M.D. ${ }^{1}$, Hojoong Kim, M.D. ${ }^{1}$, O Jung Kwon, M.D. ${ }^{1}$, Joungho Han, M.D. ${ }^{2}$, Sang-Won Um, M.D. ${ }^{1}$

In photodynamic therapy, a chemical photosensitizer is activated by light of a specific wavelength., Photodynamic therapy has been combined with bronchoscopy in patients who are ineligible for surgical resection in order to treat patients with early central lung cancer. Here, we describe 3 patients with early central lung cancer who were treated effectively using photodynamic therapy. Our experience shows that this approach is a useful modality in the therapy of these types of tumors.

Key Words: Photodynamic therapy, Early central lung cancer, Rigid bronchoscopy

\section{Introduction}

Early central lung cancer (ECLC) is generally considered to be a localized neoplastic lesion confined to the bronchial lumen and wall without lymph node metastasis ${ }^{1}$. Although the standard treatment for ECLC is surgical resection, the patient with marginal cardiopulmonary reserve must be treated non-surgically. However, of the various non-surgical treatment options, neither radiotherapy nor chemotherapy is sufficiently target-oriented for these localized cancers, and both approaches can lead to complications, including the deterioration of pulmonary function.

Following the first reported use of photodynamic therapy (PDT) for the treatment of ECLC, by Kato in $1980^{2}$, its efficacy for the treatment of this disease has been confirmed in many publications. Although PDT is currently considered to be a first-line modality as an al-

Address for correspondence: Sang-Won Um, M.D.

Division of Pulmonary and Critical Care Medicine, Department of Medicine, Samsung Medical Center, Sungkyunkwan University School of Medicine, 50, Irwon-dong, Kangnamgu, Seoul 135-710, Korea

Phone: 82-2-3410-3429, Fax: 82-2-3410-3849

E-mail: sangwonum@skku.edu

Received: Sep. 5, 2009

Accepted: Oct. 5, 2009 ternative to surgical resection ${ }^{3}$, few reports have described its use in Korea for the treatment of ECLC. Here, we describe our successful experience in the use of PDT for the management of patients with ECLC.

\section{Case Report}

\section{Patient 1}

A 76-year-old man with a 75-pack-year history of smoking presented with gross hematuria and hemoptysis. In another hospital, transurethral resection of the bladder was performed for transitional cell carcinoma of the bladder. In addition, sputum cytology revealed malignant cells. The patient was transferred to the Samsung Medical Center, where he underwent a computed tomography (CT) scan of the chest, which revealed only emphysema in the upper lung zones. On bronchoscopic examination, mucosal nodularity was shown around the orifice of the anterior segment of the right upper lobe (Figure 1A), and forceps biopsy demonstrated squamous cell carcinoma (Figure 1B). Initially, surgical resection for lung cancer was considered but the patient refused to receive surgical resection due to his old age. Therefore we performed PDT as an alternative modality to surgical resection. 
The patient was administered PDT for the treatment of ECLC using rigid bronchoscopy under general anesthesia. A hematoporphyrin derivative (Photogem ${ }^{\circledR}$; Moscow State Academy, Moscow, Russia) was administered intravenously at a dose of $2.0 \mathrm{mg} / \mathrm{kg} 48$ hours prior to PDT. A $3 \mathrm{~cm}$ cylindrical fiber (Biolitec, Jena, Germany, wavelength; $630 \mathrm{~nm}$ ) was then used to deliver laser light $(240 \mathrm{~J} / \mathrm{cm})$, irradiating the anterior and posterior segments of right upper lobe. No immediate complications occurred following PDT. Forty days later, follow-up bronchoscopic examination showed decreased mucosal irregularity and fibrotic changes (Figure 1C), and forceps biopsy revealed chronic inflammation with fibrosis (Figure 1D). No evidence of cancer recurrence was observed 25 months after PDT, and the patient was still alive at the time of this report.

\section{Patient 2}

A 59-year-old man with a 60-pack-year history of smoking presented with cough of 8 months duration. The CT scan of the chest revealed a soft-tissue mass obstructing the basal trunk of the right lower lobe and leading to atelectasis of the right lower lobe and to enlarged lymph nodes in the ipsilateral hilum, subcarinal area, and right paratracheal area. On bronchoscopic examination, the opening of right lower lobe was observed to be completely obstructed by a polypoid mass. Moreover, a small nodule was detected in the opening of right upper lobe bronchus (Figure 2A). Forceps biopsy showed squamous cell carcinoma in these two lesions (Figure 2B). The patient was diagnosed with synchronous multiple primary lung cancer because he had
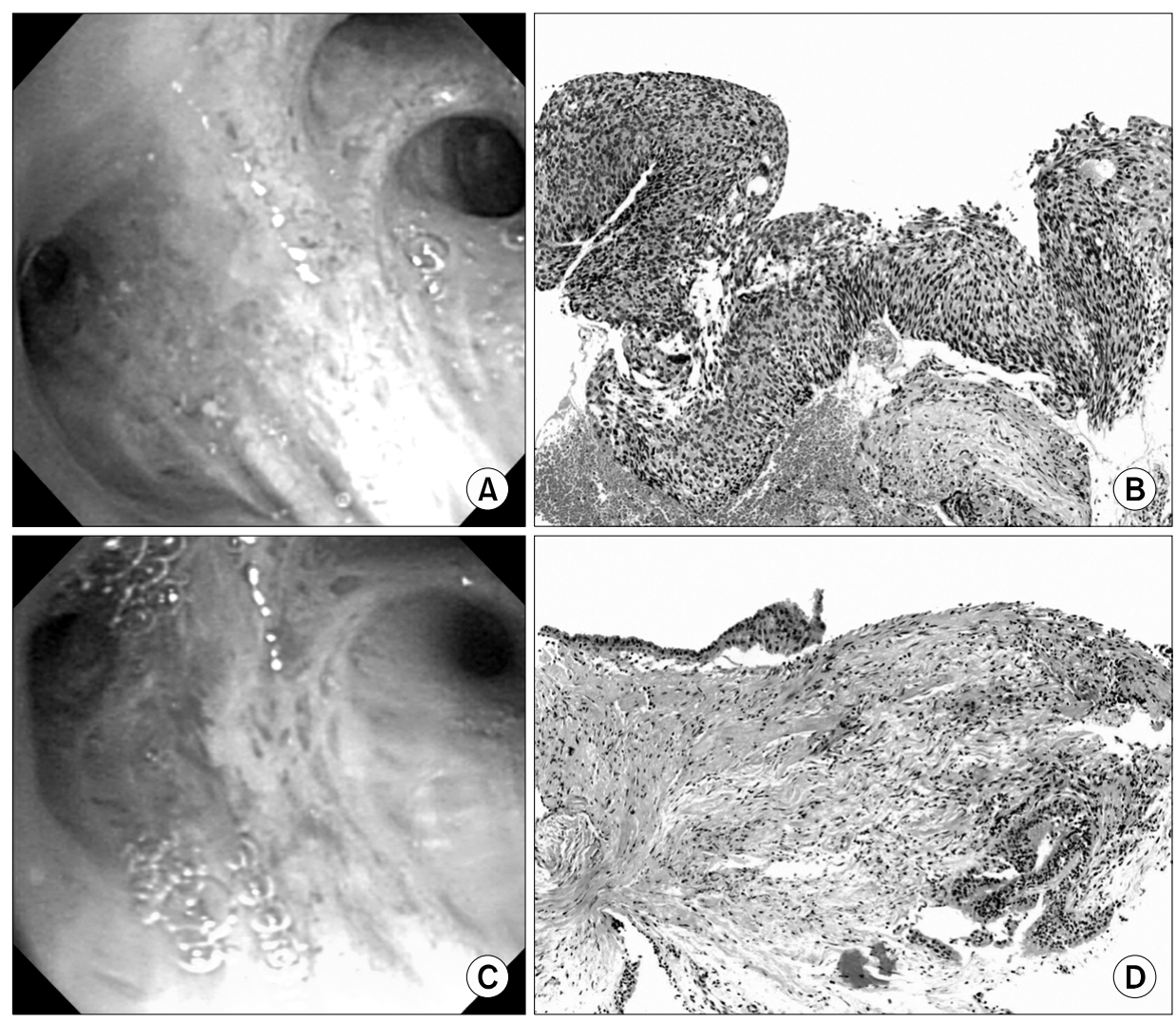

Figure 1. Patient 1, with superficial squamous cell carcinoma at the right upper lobe. (A) Bronchoscopic finding before photodynamic therapy (PDT): mucosal nodularity was shown around the orifice of the anterior segment of the right upper lobe (H\&E stain, $\times 100)$. (B) Histologic examination before PDT: squamous cell carcinoma (H\&E stain, $\times 100)$. (C) Bronchoscopic finding after PDT: decreased mucosal irregularity and fibrotic changes observed at the same site (H\&E stain, $\times 100)$. (D) Histologic examination after PDT: chronic inflammation with fibrosis (H\&E stain, $\times 100)$. 


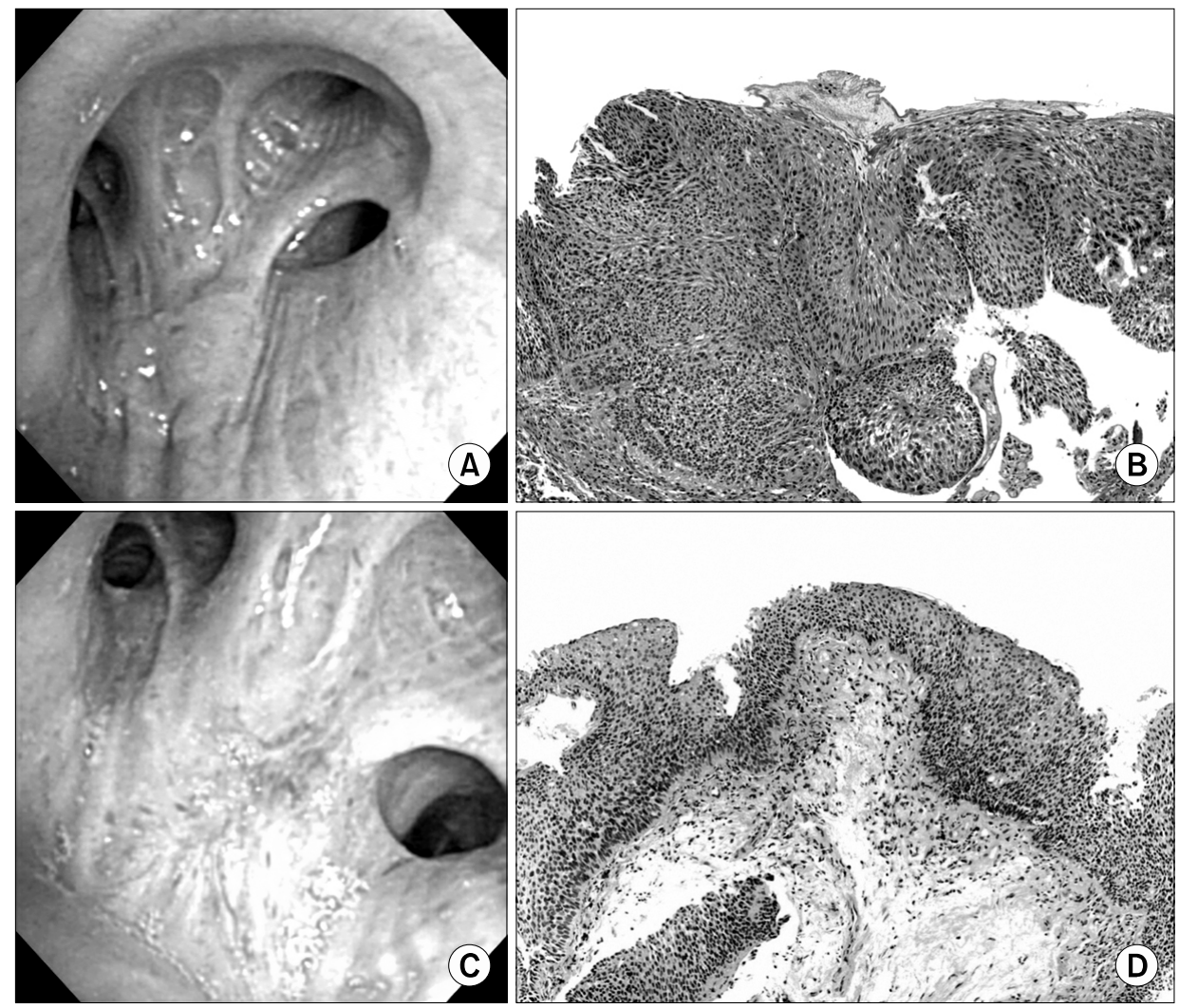

Figure 2. Patient 2, with superficial squamous cell carcinoma at the right upper lobe. (A) Bronchoscopic finding before photodynamic therapy (PDT): a small nodule was found on the opening of the right upper lobe bronchus (H\&E stain, $\times 100$ ). (B) Histologic examination before PDT: squamous cell carcinoma (H\&E stain, $\times 100)$. (C) Bronchoscopic finding after PDT: only fibrotic changes were observed at the same site (H\&E stain, $\times 100)$. (D) Histologic examination after PDT: dysplastic squamous epithelium (H\&E stain, $\times 100)$.

tumors with similar histology arising in different lobes without N2 or N3 involvement and systemic metastases. Due to marginal cadriopulmonary reserve, the patient did not seem to tolerate right pneumonectomy. Therefore we decided to perform PDT and right lower bilobectomy instead of right peumonectomy.

Firstly, PDT was administered for the right upper lobe lesion during rigid bronchoscopy under general anesthesia. A hematoporphyrin derivative was administered intravenously at a dose of $2.0 \mathrm{mg} / \mathrm{kg} 48$ hours prior to PDT. A $3 \mathrm{~cm}$ cylindrical fiber was then used to deliver laser light $(240 \mathrm{~J} / \mathrm{cm})$ for PDT, irradiating the right upper lobe bronchus. No immediate complications occurred.

After two weeks, he underwent a right lower bilobectomy with mediastinal lymph node dissection. The pathologic stage of the tumor was graded as T1N1M0.
The patient subsequently received four cycles of adjuvant chemotherapy with paclitaxel and carboplatin.

One month after PDT, only fibrotic changes were seen in the right upper lobe on follow-up bronchosopic examination (Figure 2C). Forceps biopsy showed only dysplastic squamous epithelium (Figure 2D). No evidence of cancer recurrence was observed on follow-up CT scan of the chest after 23 months, and the patient was still alive at the time of this report.

\section{Patient 3}

A 70-year-old man with a 40-pack-year history of smoking presented with a small nodule that had been detected on a routine chest x-ray. He was asymptomatic, and his physical examination was unremarkable. The CT scan of the chest revealed a $20 \mathrm{~mm}$ nodule in 


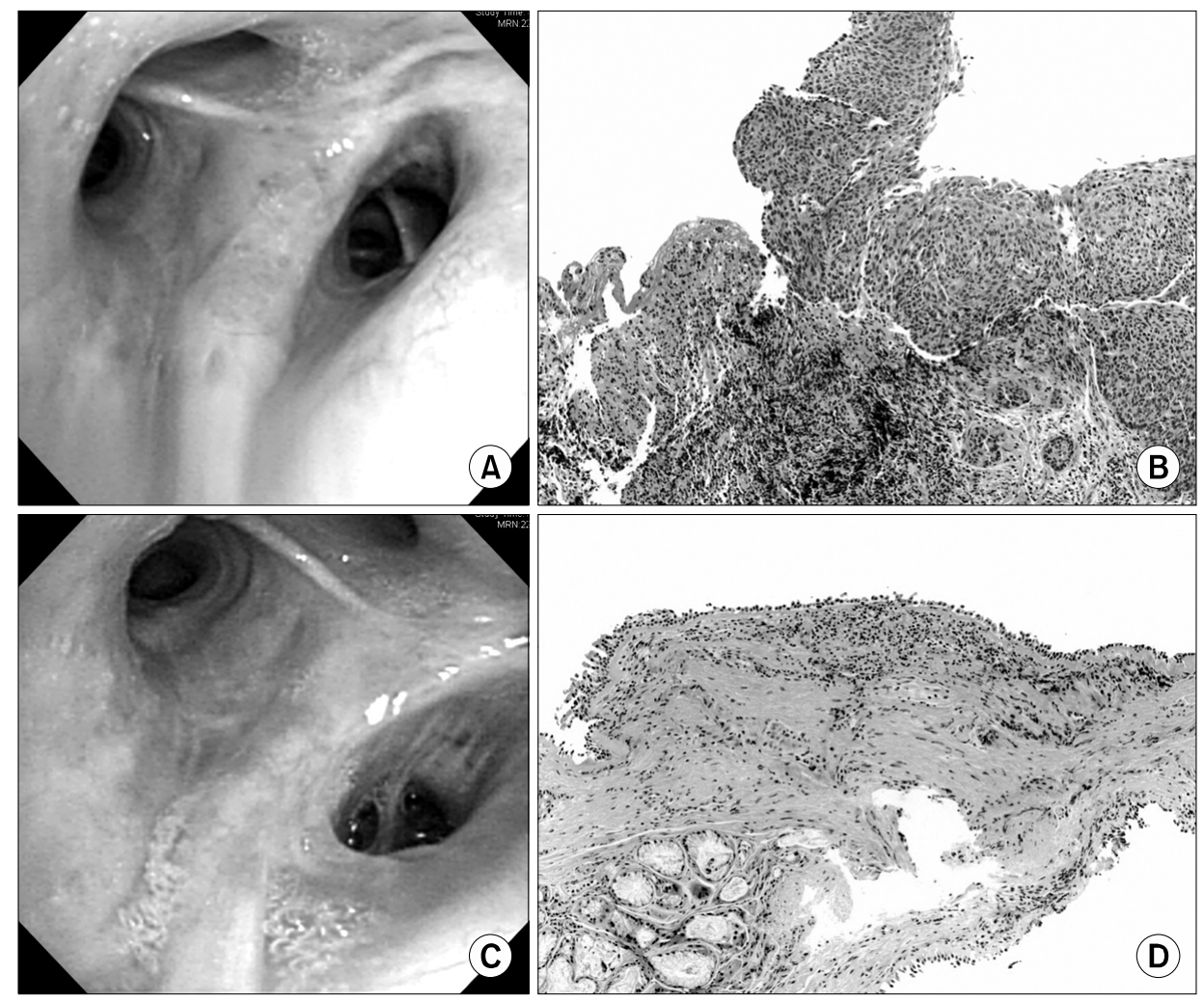

Figure 3. Patient 3, with superficial squamous cell carcinoma at the right upper lobe. (A) Bronchoscopic finding before photodynamic therapy (PDT): a mucosal nodule was shown on the bifurcation of the anterior and posterior segments of the right upper lobe (H\&E stain, $\times 100)$. (B) Histologic examination before PDT: squamous cell carcinoma (H\&E stain, $\times 100)$. (C) Bronchoscopic finding after PDT: fibrotic changes were seen at the same site (H\&E stain, $\times 100)$. (D) Histologic examination after PDT: chronic inflammation with fibrosis (H\&E stain, $\times 100)$.

the right lower lobe, with underlying pulmonary fibrosis exhibiting a typical interstitial pneumonitis pattern. On bronchoscopic examination, a mucosal nodule was detected at the bifurcation of the anterior and posterior segments of the right upper lobe (Figure 3A). Forceps biopsy revealed squamous cell carcinoma (Figure 3B). No endobronchial lesion was observed on the right lower lobe.

Pulmonary function test showed mild obstructive pattern, and echocardiogram revealed systolic dysfunction with ejection fraction of $44 \%$ and regional wall motion abnormalities. Due to marginal cadriopulmonary reserve, the patient did not seem to tolerate right pneumonectomy.

The patient underwent a lobectomy for the nodule on the right lower lobe, and the tumor histology was consistent with large-cell neuroendocrine carcinoma of pathologic stage T2NOMO, the patient received PDT for the treatment of squamous cell carcinoma of the right upper lobe using rigid bronchoscopy under general anesthesia. A hematoporphyrin derivative was administered intravenously at a dose of $2.0 \mathrm{mg} / \mathrm{kg} 48$ hours prior to PDT. A $3 \mathrm{~cm}$ cylindrical fiber was used to deliver laser light $(220 \mathrm{~J} / \mathrm{cm})$, irradiating the right upper lobe bronchus.

Three weeks after PDT, four cycles of adjuvant chemotherapy with etoposide and cisplatin were administered for large-cell neuroendocrine carcinoma of the right lower lobe. Follow-up bronchoscopy after 3 months revealed fibrotic changes at the PDT site (Figure 3C) that had resulted in chronic inflammation, as demonstrated by forceps biopsy (Figure 3D). No evidence 
of local tumor recurrence or metastatic lesion in the thorax was found on chest CT scan after 17 months, and the patient was still alive at the time of this report.

\section{Discussion}

Photodynamic therapy was first studied in Japan experimentally in 1978 and applied clinically for the treatment of lung cancer in $1980^{2}$. It is currently approved by both the Food and Drug administration and European Agencies as a treatment option for patients with ECLC who are ineligible for surgical resection and for the palliation of airway obstruction caused by malignant tumors.

The principle of PDT in the treatment of lung cancer is as follows. A photosensitizing agent is injected, and after a suitable time interval, tumor tissue that has retained the compound is exposed to laser light of 630 nm wavelength. The laser is delivered using a fiber introduced through the bronchoscope, and the resulting nonthermal phototoxic reaction leads to delayed cell death. Follow-up bronchoscopy is necessary to remove debris and secretions. The main adverse effect of this approach is associated skin photosensitivity, which can last up to 6 weeks.

Phase I-II clinical trials have shown that, in ECLC, complete eradication of the tumor can be achieved in about $90 \%$ of these patients, with disease-free intervals of up to 11 years ${ }^{4}$. In patients with superficial extension of the tumor at a central airway, preoperative PDT can convert an unresectable tumor to a resectable state or reduce the extent of surgery from a pneumonectomy to a sleeve lobectomy or bilobectomy ${ }^{5}$. Kato et $\mathrm{al}^{6}$ reported that PDT achieved an 80\% complete response rate among 35 patients with bronchoscopically proven ECLC. Three of these patients received PDT alone and survived more than 5 years. Edell and Cortese ${ }^{7}$ performed PDT as an alternative to surgery in patients with early superficial cancer who were eligible for surgery. Of 14 cancers, $71 \%$ showed a complete response after a single course of PDT, while $21 \%$ required a second course of PDT to achieve a complete response. Endo et $\mathrm{al}^{8}$ reported the results of long-term follow up of PDT for roentgenographically occult bronchogenic squamous cell carcinoma. In their 48 patients, a complete response was achieved in $94 \%$, but $20 \%$ had local recurrence after complete response. Five- and ten-year overall survival rates for all 48 patients were, respectively, $81 \%$ and $71 \%$.

In Korea, however, only two reports have described the use of PDT for palliative treatment in patients with advanced lung cancer ${ }^{9,10}$, and only one report addressed the use of PDT in the treatment of ECLC ${ }^{11}$. The two patients described in the latter were successfully treated, one for local recurrence at a previous pneumonectomy site and the other for metachronous multiple primary lung cancer ${ }^{11}$. Our three patients with ECLC who were treated with PDT differed from those described in that report. One patient had a double primary tumor related to bladder cancer and the other two patients were diagnosed with synchronous multiple primary lung cancer. A complete response was achieved in all three patients.

Recently, endobronchial ultrasonography (EBUS) and autofluorescence bronchoscopy (AFB) have been reported as new modalities for use in the selection of patients with ECLC who are appropriate candidates for PDT with curative intent. AFB allows evaluation of the endobronchial extension of the tumor and EBUS of the perpendicular extension of the tumor into the bronchial wall. A tumor diagnosed as intracartilaginous by EBUS can be successfully treated with $\mathrm{PDT}^{12}$. With respect to tumor size, fewer than $10 \%$ of tumors $<2.0 \mathrm{~cm}$ have lymph node metastasis, based on the evaluation of surgical specimens ${ }^{13}$. In addition, one report suggested that for bronchoscopically superficial tumors with a surface diameter $<1.0 \mathrm{~cm}$ or nodular tumors with a surface diameter $<0.5 \mathrm{~cm}$, complete remission can be achieved with PDT $^{14}$.

For porphyrin-based PDT, we have used light doses of $220 \sim 240 \mathrm{~J} / \mathrm{cm}$ administered through a diode laser system emitting red light at $630 \mathrm{~nm}$. Although some reports in the literature describe the use of light doses of $150 \sim 300 \mathrm{~J} / \mathrm{cm}$ for illumination of the lesion ${ }^{15}$, currently, the majority recommend a light dose of about $200 \mathrm{~J} / \mathrm{cm}$ 
of tumor for porphyrin-based PDT in bronchoscopic applications. However, Endo et al reported that a complete response to PDT was achieved in 94\% of their ECLC patients using laser irradiation with a total energy of $100 \mathrm{~J} / \mathrm{cm}^{2}$. Therefore, the total energy of the laser used by this group is smaller than that recommended in previous studies ${ }^{8}$. Nonetheless, following the delivery of relatively high doses of energy, our patients with ECLC achieved a complete response, and no complications were observed. Clearly, additional studies are needed in which both the tumor response and the complications following PDT administered with low vs. high doses of laser irradiation energy are evaluated.

It should also be noted that we performed PDT using rigid rather than flexible bronchoscopy. Although the latter technique has the advantages of topical anesthesia and sedation, we prefer rigid bronchoscopy as it allows fixation of the optical fiber around the cancer tissue, easier bronchial tree cleaning, greater inhibition of cough, and greater sedation, all of which contribute to safer and more successful treatment than is obtained using flexible bronchoscopy.

The combined effect of PDT and chemotherapy is not well known in patients with primary lung cancer. In this report, two patients receive PDT and adjuvant chemotherapy. There is a possibility that adjuvant chemotherapy contributes to disease-free survival. In fact, chemotherapy and/or radiation therapy were used in 32\% of patients with esophageal cancer to supplement primary $\mathrm{PDT}^{16}$. Moreover, the antitumor effect of PDT was enhanced by administration of cisplatin before PDT in animal model ${ }^{17}$. In the future, further studies are required to evaluate the benefit of integrating PDT with radiation and chemotherapy.

However, this report has two limitations. The first is the relatively short follow up of all three patients (25, 23, and 17 months). The second is the small number of patients who underwent PDT for the treatment of ECLC. Clearly, more experience with PDT in the treatment of ECLC and longer follow up of patients are required.

In conclusion, this report describes the cases of three patients with early central lung cancer who were treated effectively using PDT. We believe that PDT is a useful modality in the treatment of early central lung cancer.

\section{References}

1. Moghissi K, Dixon K, Thorpe JA, Stringer M, Oxtoby C. Photodynamic therapy (PDT) in early central lung cancer: a treatment option for patients ineligible for surgical resection. Thorax 2007;62:391-5.

2. Kato H. Photodynamic therapy for lung cancer: a review of 19 years' experience. J Photochem Photobiol B 1998;42:96-9.

3. Kennedy TC, McWilliams A, Edell E, Sutedja T, Downie G, Yung R, et al. Bronchial intraepithelial neoplasia/ early central airways lung cancer: ACCP evidence-based clinical practice guidelines (2nd edition). Chest 2007; 132:221S-33S.

4. Kato H, Kawate N, Kinoshita K, Yamamoto H, Furukawa K, Hayata Y. Photodynamic therapy of early-stage lung cancer. Ciba Found Symp 1989;146:183-97.

5. Kato H, Konaka C, Ono J, Kawate N, Nishimiya K, Shinohara $\mathrm{H}$, et al. Preoperative laser photodynamic therapy in combination with operation in lung cancer. J Thorac Cardiovasc Surg 1985;90:420-9.

6. Kato H, Konaka C, Kawate N, Shinohara H, Kinoshita K, Noguchi M, et al. Five-year disease-free survival of a lung cancer patient treated only by photodynamic therapy. Chest 1986;90:768-70.

7. Edell ES, Cortese DA. Photodynamic therapy in the management of early superficial squamous cell carcinoma as an alternative to surgical resection. Chest 1992; 102:1319-22.

8. Endo C, Miyamoto A, Sakurada A, Aikawa H, Sagawa M, Sato M, et al. Results of long-term follow-up of photodynamic therapy for roentgenographically occult bronchogenic squamous cell carcinoma. Chest 2009;136: 369-75.

9. Yoon SH, Han KT, Kim GN, Lee SI. Effect of photodynamic therapy in lung cancer. Tuberc Respir Dis 2004;57:358-63.

10. Kim YK, Lee YM, Kim KU, Uh ST, Kim YH, Park CS. Clinical experience of photodynamic therapy in five patients with advanced lung cancer. Tuberc Respir Dis 2004;57:72-7.

11. Jang TW, Kim HK, Oak CH, Jung MH. Photodynamic therapy in early lung cancer: A report of two cases. Korean J Intern Med 2006;21:178-82.

12. Miyazu Y, Miyazawa T, Kurimoto N, Iwamoto Y, Kan- 
$\mathrm{JH}$ Lee et al: Usefulness of PDT in the management of early central lung cancer

oh K, Kohno N. Endobronchial ultrasonography in the assessment of centrally located early-stage lung cancer before photodynamic therapy. Am J Respir Crit Care Med 2002;165:832-7.

13. Saito Y, Nagamoto N, Ota S, Sato M, Sagawa M, Kamma K, et al. Results of surgical treatment for roentgenographically occult bronchogenic squamous cell carcinoma. J Thorac Cardiovasc Surg 1992;104:401-7.

14. Kato H, Okunaka T, Shimatani H. Photodynamic therapy for early stage bronchogenic carcinoma. J Clin Laser Med Surg 1996;14:235-8.

15. Moghissi K, Dixon K. Update on the current indic- ations, practice and results of photodynamic therapy (PDT) in early central lung cancer (ECLC). Photodiagnosis Photodyn Ther 2008;5:10-8.

16. Keeley SB, Pennathur A, Gooding W, Landreneau RJ, Christie NA, Luketich J. Photodynamic therapy with curative intent for Barrett's esophagus with high grade dysplasia and superficial esophageal cancer. Ann Surg Oncol 2007;14:2406-10.

17. Uehara M, Inokuchi T, Ikeda H. Enhanced susceptibility of mouse squamous cell carcinoma to photodynamic therapy combined with low-dose administration of cisplatin. J Oral Maxillofac Surg 2006;64:390-6. 